Research, Society and Development, v. 10, n. 15, e381101522670, 2021

(CC BY 4.0) | ISSN 2525-3409 | DOI: http://dx.doi.org/10.33448/rsd-v10i15.22670

\title{
Percepções de puérperas acerca da violência obstétrica em uma maternidade de
}

\author{
Manaus \\ Perceptions of postpartum women about obstetric violence in a maternity hospital in Manaus \\ Percepciones de las puérperas sobre la violencia obstétrica en una maternidad de Manaus
}

Recebido: 02/11/2021 | Revisado: 09/11/2021 | Aceito: 17/11/2021 | Publicado: 24/11/2021

\author{
Carina dos Santos Goes \\ ORCID: https://orcid.org/0000-0002-0088-0381 \\ Centro Universitário FAMETRO, Brasil \\ E-mail: carina.goes.1998.cg@gmail.com \\ Jéssica de Souza Almeida \\ ORCID: https://orcid.org/0000-0003-3587-363X \\ Centro Universitário FAMETRO, Brasil \\ E-mail: jessica.dsa2016@gmail.com \\ Priscila Alves da Silva \\ ORCID: https://orcid.org/0000-00027198-4153 \\ Centro Universitário FAMETRO, Brasil \\ E-mail: priscilaalves.silvaa30@gmail.com \\ Graciana de Sousa Lopes \\ ORCID: https://orcid.org/0000-0003-3615-9040 \\ Centro Universitário FAMETRO, Brasil \\ E-mail: gracilopess@hotmail.com \\ Monike Emyline Andrade Rodrigues \\ ORCID: https://orcid.org/0000-0001-7607-7854 \\ Centro Universitário FAMETRO, Brasil \\ E-mail: emyline_monike@hotmail.com \\ Andreza Mendes dos Santos \\ ORCID: https://orcid.org/0000-0002-8375-1218 \\ Centro Universitário FAMETRO, Brasil \\ E-mail: gracilopess@hotmail.com
}

\begin{abstract}
Resumo
Objetivo: investigar as percepções das puérperas acerca da Violência Obstétrica em uma Maternidade de Manaus. Metodologia: pesquisa de campo, transversal, descritiva, exploratória com abordagem qualitativa. Foram selecionadas para entrevistas puérperas que haviam passado por Parto Normal (PN) 24 horas pós-parto ou Parto Cesáreo (PC) em uma maternidade de referência na cidade de Manaus- AM. A amostragem foi elaborada através de Saturação Teórica, com 31 participantes. Pesquisa aprovada pelo Comitê de Ética em Pesquisa (CAAE: 95471018.8.0000.0004). Resultados: a violência obstétrica está relacionada à condições sociodemográficas desfavoráveis, a falta de conhecimento e baixa escolaridade, o que desencadeia a dificuldade em identificar a sua ocorrência. Observou-se violência verbal, toques vaginais sem indicação, manobra de Kristeller, entre outras. Além disso, percepções de ansiedade, medo e desrespeito. Conclusão: com o avanço das boas práticas no parto, observa-se a diminuição de casos de violência obstétrica, porém ainda há muito a ser desenvolvido. A Enfermagem é protagonista neste processo de desenvolvimento da humanização do parto e proteção da autonimia e direitos da gestante.
\end{abstract}

Palavras-chaves: Violência Obstétrica; Puerperal; Grávida; Parto.

\section{Summary}

Objective: to investigate the perceptions of postpartum women about Obstetric Violence in a Maternity Hospital in Manaus. Methodology: field, cross-sectional, descriptive, exploratory research with a qualitative approach. They were selected for interviews in puerperal women who had undergone Normal Delivery $(\mathrm{PN}) 24$ hours postpartum or Cesarean delivery (CP) in a reference maternity hospital in the city of Manaus-AM. The sampling was elaborated through Theoretical Saturation, with 31 participants. Research approved by the Research Ethics Committee (CAAE: 95471018.8.0000.0004). Results: obstetric violence is related to unfavorable sociodemographic conditions, lack of knowledge and low education, which triggers the difficulty in identifying its occurrence. Verbal violence, vaginal touching without indication, Kristeller maneuver, among others, were observed. Also, perceptions of anxiety, fear and disrespect. Conclusion: with the advancement of good practices in childbirth, there is a reduction in cases of obstetric 
violence, but there is still much to be developed. Nursing is the protagonist in this process of developing the humanization of childbirth and protecting the autonomy and rights of pregnant women.

Keywords: Obstetric Violence; Puerperal; Pregnant; Parturition.

\begin{abstract}
Resumen
Objetivo: investigar las percepciones de las puérperas sobre la Violencia Obstétrica en una Maternidad de Manaus. Metodología: investigación de campo, transversal, descriptiva, exploratoria con enfoque cualitativo. Fueron seleccionadas para entrevistas en puérperas que habían sido sometidas a Parto Normal (NP) 24 horas posparto o Parto por cesárea $(\mathrm{CP})$ en una maternidad de referencia en la ciudad de Manaus-AM. El muestreo se elaboró mediante Saturación Teórica, con 31 participantes. Investigación aprobada por el Comité de Ética en Investigación (CAAE: 95471018.8.0000.0004). Resultados: la violencia obstétrica se relaciona con condiciones sociodemográficas desfavorables, desconocimiento y baja escolaridad, lo que desencadena la dificultad para identificar su ocurrencia. Se observaron violencia verbal, toques vaginales sin indicación, maniobra de Kristeller, entre otros. Además, percepciones de ansiedad, miedo y falta de respeto. Conclusión: con el avance de las buenas prácticas en el parto, hay una reducción de los casos de violencia obstétrica, pero aún queda mucho por desarrollar. La enfermería es la protagonista de este proceso de desarrollo de la humanización del parto y protección de la autonomía y los derechos de la gestante.
\end{abstract}

Palabras clave: Violencia obstétrica; Puerperal; Embarazada; Parto

\title{
1. Introdução
}

A violência obstétrica (VO) vem sendo alvo de grande debate em ralação as condutas dos profissionais de saúde na assistência ao parto, pois esses atos não atingem somente a parturiente, mas também os familiares e os próprios recém-nascidos (Ramos, 2020). E mesmo com um alto índice a VO ainda é um assunto que continua sendo um tabu por ser uma questão delicada que envolve tanto profissionais de saúde como a ineficiência de políticas públicas de combate a tais práticas (Silva, 2019).

Nota-se que a falta de conhecimento sobre a violência obstétrica é um dos fatores que contribuem para o alto índice desse tipo de violência, pois as mulheres que estão em trabalho de parto estão em um momento de muita vulnerabilidade emocional causado pelos hormônios da gravidez e a tensão que o momento do parto ocasiona. Assim, o momento que deveria ficar marcado na vida da mulher como um dia de felicidade, acaba ficando marcado como um dia de violações (Souza; Mangueira; Alexandre,2020).

A violência obstétrica mostra uma ótica negativa do parto, influenciando a percepção das mulheres que acabam aceitando aquilo que é proposto pelos profissionais, pois as gestantes não possuem o conhecimento do que é violência obstétrica. A ocorrência da VO está ligada a práticas arcaicas e se dão com mais frequência na população de baixa renda, refletindo a falta de humanização na assistência ao parto e nascimento (Lansky et al., 2019).

Para que eles não precisem passar por um parto longo e extremamente exaustivo, os profissionais optam por procedimentos que não são adequados e que acabam colocando a vida da mulher e a vida do recém-nascido em risco. Muitas vezes descumprindo as recomendações propostas pelo Ministério da Saúde e OMS. (Zanardo et al., 2017).

Toda a mulher é protegida por lei a usufruir de uma assistência digna e de qualidade na hora do parto, porém esses direitos acabam não sendo respeitados. Dentre eles o direito ao acompanhante, amparado pela Lei de $\mathrm{n}^{\circ} 11.108$, que garante a parturiente ter um acompanhante de sua escolha. Além da autonomia de decidir em quais posições ela se sente mais confortável e confiante em parir como: quatro apoios, cócoras, sentada em banqueta, lateral, em pé, entre outras (Coelho e Marques, 2016).

Em primeiro plano, é sugerido o parto normal, pois, é mais proveitoso e seguro para a mãe e seu bebê. Em segundo plano o parto cesariano, sendo utilizado apenas em situações no qual o parto normal oferece risco. Ao contrário do que é estabelecido pelas políticas de saúde os números de partos por cesariana acabam superando os de parto natural, ainda que sejam estimuladas por campanhas, acabam optando pela segunda escolha que na maioria das vezes são desnecessárias, e perdem o direito de desfrutar dos benefícios do parto normal (Vicente; Lima; Lima, 2017). 
As gestantes com mais acesso a informação, entendem que é possível realizar mínimas intervenções durante seu processo de parto, respeitando suas dores e seus limites. Técnicas para ajudar seu processo devem ser explanadas durante o prénatal, como respiração correta, massagens na hora das dores, exercícios que fortalecem os músculos do períneo, aromaterapia, bola suíça, banho de água morna entre outros, ajudando no alivio da dor e na fase de expulsão do seu momento de parir. Porém, essas técnicas são pouco utilizadas nos atendimentos em maternidades, devido à falta de recursos e capacitação dos profissionais de saúde (Araújo; Andrade; Vasconcelos, 2020).

As mulheres necessitam de uma melhor compreensão sobre violência obstétrica durante a gestação, compreensão esta que podem ser estabelecidas por meio de educação em saúde, atividades educativas e melhoria na qualidade do pré-natal. Tendo essas informações sobre seus direitos e o processo fisiológico do seu corpo, elas se diferenciam ao adentrar na maternidade quando se refere à violência obstétrica (Nascimento et al., 2019).

Os profissionais necessitam de atualização para atuarem em sua área, pois é de sua responsabilidade passar informações com baseamento científico para as parturientes, incluindo ações humanizadas, empatia ao realizar os cuidados, e respeitar seus direitos estabelecidos por leis (Campos et al., 2020).

É necessário a orientação, capacitação dos profissionais de saúde, além de implementação de protocolos para prevenção à violência obstétrica para que as parturientes venham ter uma assistência adequada e digna no parto e nascimento. E faz-se necessário a criação de políticas eficazes para a identificação e combate da VO (Castro e Rocha, 2020).

$\mathrm{O}$ acesso a informação à gestantes se faz necessário durante todo seu período gestacional, onde a mesma passa a compreender seu corpo e seus direitos que irão ocorrer durante esse processo (Carneiro, 2021). Diante disso, a seguinte pesquisa tem por objetivo investigar as percepções das puérperas acerca da Violência Obstétrica em uma Maternidade de Manaus, e inquirir os tipos de violência obstétrica mais incidentes.

\section{Metodologia}

Trata-se de uma pesquisa de campo, descritiva, exploratória, transversal, com abordagem qualitativa. A abordagem qualitativa trabalha questões subjetivas, parte do fundamento de que há uma relação dinâmica entre o mundo real e o sujeito, uma interdependência viva entre o sujeito e o objeto, um vínculo indissociável entre o mundo objetivo e a subjetividade do sujeito, buscando interpretar fenômenos multifatoriais (Piana, 2009).

A pesquisa foi desenvolvida em 3 momentos, primeira: entrevista na maternidade, segundo: categorização dos resultados e terceiro: análise de conteúdo.

Foram selecionadas puérperas que tenham passado por Parto Normal (PN) 24 horas pós-parto ou Parto Cesáreo (PC) em uma maternidade de referência na cidade de Manaus- AM. A amostragem foi selecionada através de Saturação Teórica, com 31 participantes. Considera-se saturada a coleta de dados quando nenhum novo elemento é encontrado e o acréscimo de novas informações deixa de ser necessário, pois não altera a compreensão do fenômeno estudado. Trata-se de um critério que permite estabelecer a validade de um conjunto de dados (Rhiry- Cherques, 2009).

Os Critérios de Elegibilidade foram: mulheres que tenham sido atendidas na maternidade selecionada para a pesquisa, maiores de 18 anos, que tenham ido à, pelo menos, 4 consultas pré-natal e que aceitem participar do estudo. Os critérios de Inelegibilidade foram: puérperas de parto gemelar e que tenham algum comprometimento que às impeçam de comunicar-se.

A coleta de dados se deu através de entrevista semiestruturada, no Alojamento Conjunto (ALCON) da maternidade escolhida, com auxílio de gravador de voz. Os Instrumentos de coleta de dados utilizados foram: um Roteiro de Entrevista Semiestruturada para guiar a entrevista, já validado (adaptado de Pereira, 2014), possuindo informações do prontuário, sendo composto de duas partes, contendo perguntas abertas e fechadas e um formulário sociodemográfico. 
Considerações éticas: o estudo seguiu as diretrizes e normas regulamentadoras de pesquisas envolvendo seres humanos da resolução 466 de 12 de dezembro de 2012. E o início da coleta de dados só iniciou após autorização de Comitê de Ética. Antes da entrevista, cada puérpera foi informada sobre o estudo e convidada a participar, assinando, assim, o termo de Consentimento Livre e Esclarecido. Os participantes tiveram a autonomia de deixar de participar do estudo a qualquer momento que desejassem. A confidencialidade e o sigilo quanto aos dados coletados foram assegurados pelas pesquisadoras, sendo identificadas pela letra P (Puérperas) seguida de um algarismo arábico (P1, P2, P3..., P31), conforme a realização das entrevistas.

Os riscos dessa pesquisa foram mínimos, visto que foram aplicadas apenas entrevistas com as participantes. Os riscos possíveis, previamente identificados, poderiam ser de cunho psicológico, envolvendo expressão de tristeza, vergonha, entre outros. Deste modo, houve a preocupação com a privacidade e respeito pelos sentimentos experimentados pelas participantes, através de suporte psicológico quando necessário.

Dentre os benefícios deste estudo estão o aumento do conhecimento sobre a violência obstétrica na cidade de Manaus e a contribuição na divulgação de meios de prevenção de tal conduta.

\section{Resultados e Discussão}

O estudo evidenciou no Gráfico 1 que existe uma relação entre baixa renda, grau de escolaridade e violência obstétrica. Segundo Farias et al (2021, p 7) "puérperas são em sua maioria pardas, com idade entre 18 e 35 anos, que possuem pouca escolaridade e baixa renda familiar". e esses dados diferem de estudo de Lansky (2019), em que se observou VO com mulheres com nível elevado de escolaridade e renda. Demonstrando que a VO ocorre em todos os níveis socioeconômicos.

Gráfico 1- Renda Familiar

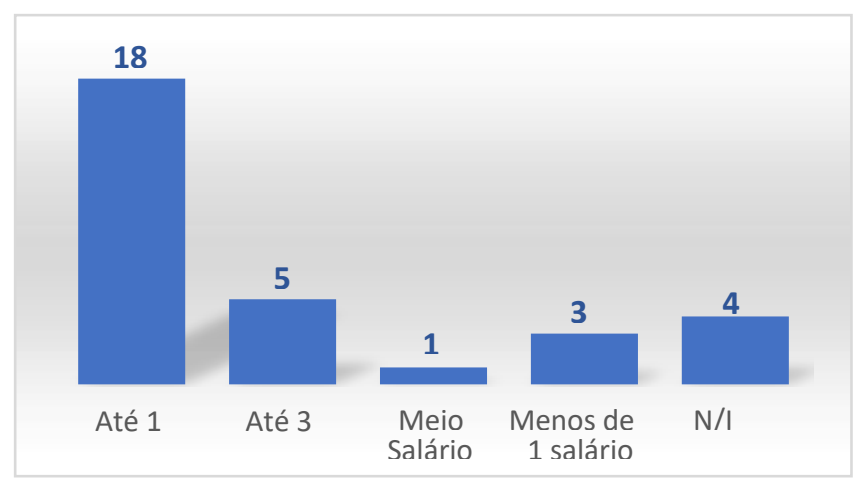

Fonte: Autores (2021).

O Gráfico 2 apresenta a porcentagem de mulheres grávidas solteiras ou em união estável em comparação às casadas. Neste contexto, infere-se que esse também é um fator que contribui com a violência obstétrica, pois está relacionada com a falta de planejamento da gravidez, o que acaba tornando-as mais vulneráveis à VO. Segundo Novellino (2011, p.312). “Ser mãesolteira implica em diferentes graus de vulnerabilidade". 
Gráfico 2 - Estado Civil.

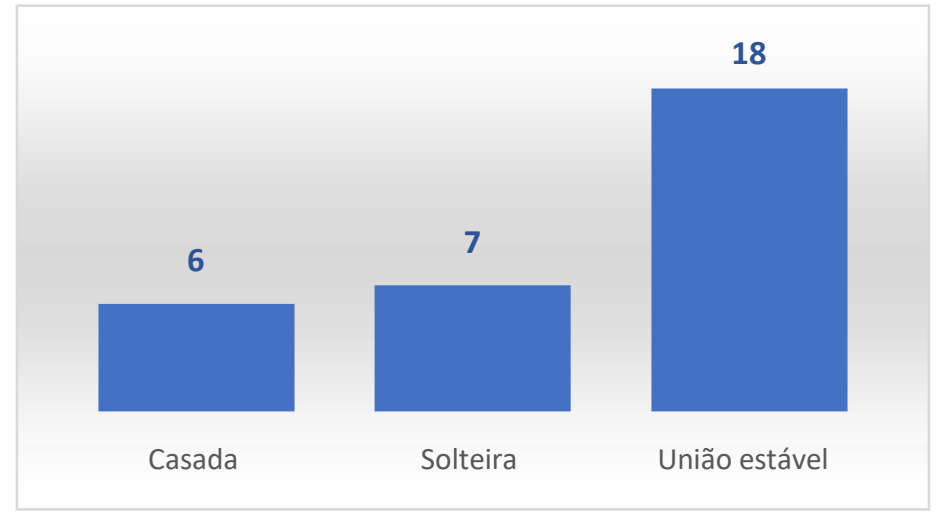

Fonte: Autores (2021).

O Gráfico 3 mostra o número de gravidez que não foram planejadas, o que pode influenciar na ocorrência de violência obstétrica, pois uma vez não planejadas essas gestações não recebem o suporte ideal durante o pré-natal. Brandão (2021). Cita que ainda se encontra um número considerável de mulheres com um alto percentual de gestações imprevistas, bem como a da reprodução em momento inoportuno. É importante que a mulher seja orientada desde o pré-natal e desenvolva vínculos com a equipe de saúde para que, assim ela tenha conhecimento dos procedimentos que serão realizados, fazendo com que tenha mais confiança na hora do parto.

Gráfico 3 - A gravidez atual foi planejada?

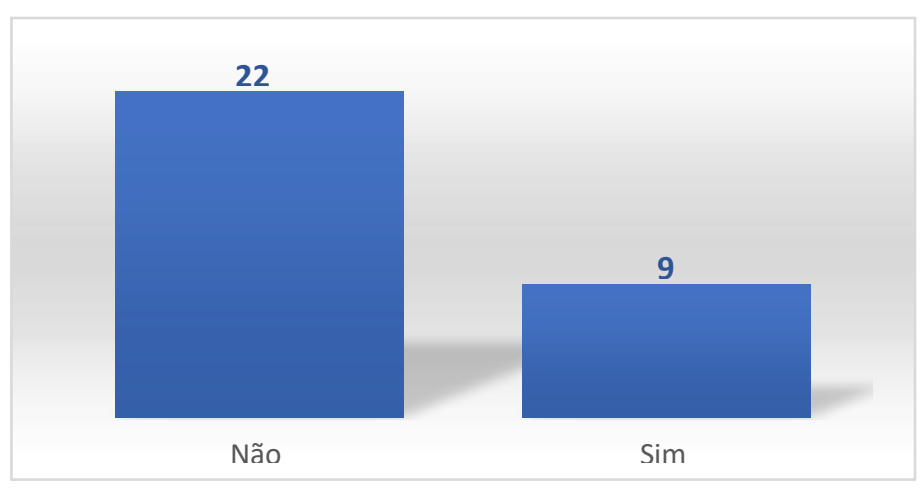

Fonte: Autores (2021).

O acompanhante é muito importante no processo parturitivo, pois é ele que proporciona apoio tanto físico quanto psicológico na hora do parto e faz com que a mulher se sinta mais segura por ter uma presença familiar. Porém, ainda é possível observar mulheres sem saber do seu direito ao acompanhante no processo de parto, negligenciando-se o direito dessas mulheres. Segundo Tomasi et al (2019). A presença de acompanhante no parto já é reconhecida como uma boa prática obstétrica, um direito garantido pela legislação, como mostra o Gráfico 4. 
Research, Society and Development, v. 10, n. 15, e381101522670, 2021

Gráfico 4 - Você teve direito ao acompanhante de sua escolha?

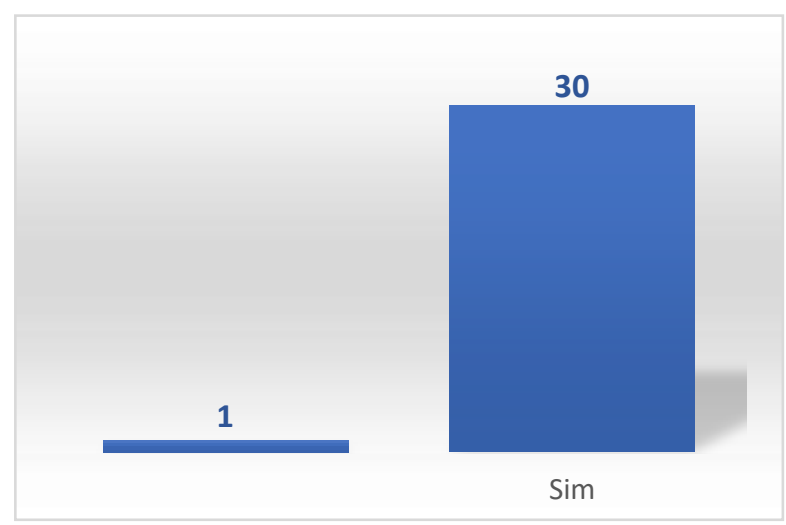

Fonte: Autores (2021).

O Gráfico 5 mostra a importância da orientação por parte da equipe de saúde sobre os tipos de procedimentos que serão realizados, pois quaisquer tipos de intervenções devem ser previamente esclarecidos e autorizados, para não serem caracterizados como violência obstétrica. Segundo Marques (2019). Devem-se definir métodos e estratégias, a fim de garantir que a gestante receba todas as orientações preconizadas e tenha possibilidade de executá-las.

Gráfico 5 - No momento do parto você foi orientado aos procedimentos a serem realizados?

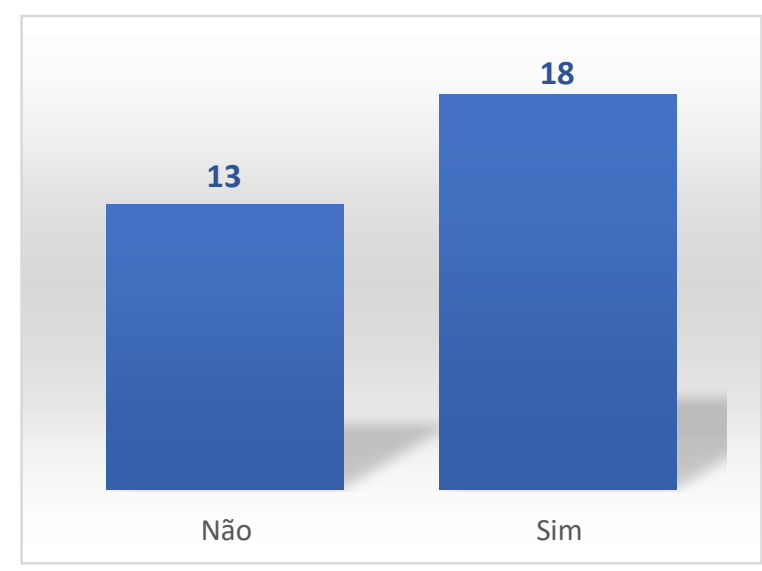

Fonte: Autores (2021).

O excesso de toques vaginais podem trazer vários riscos como: parto prematuro e aumento do risco de contaminação do feto. As mulheres não têm o conhecimento dos malefícios que o excesso desse procedimento podem acarretar, e que sem o consentimento da mesma configura-se uma violência obstétrica. De acordo com Souza et al, (2016, p.6),"Realização demasiada e sem consentimento de toques vaginais foram alguns dos casos de violência ocorridos, onde as mesmas não tinham conhecimentos de que eram atos de violência obstétrica", no Gráfico 6 é possivel observar essa realidade neste estudo. 
Gráfico 6 - Quando realizaram o exame de toque vaginal, explicaram para você o motivo da realização?

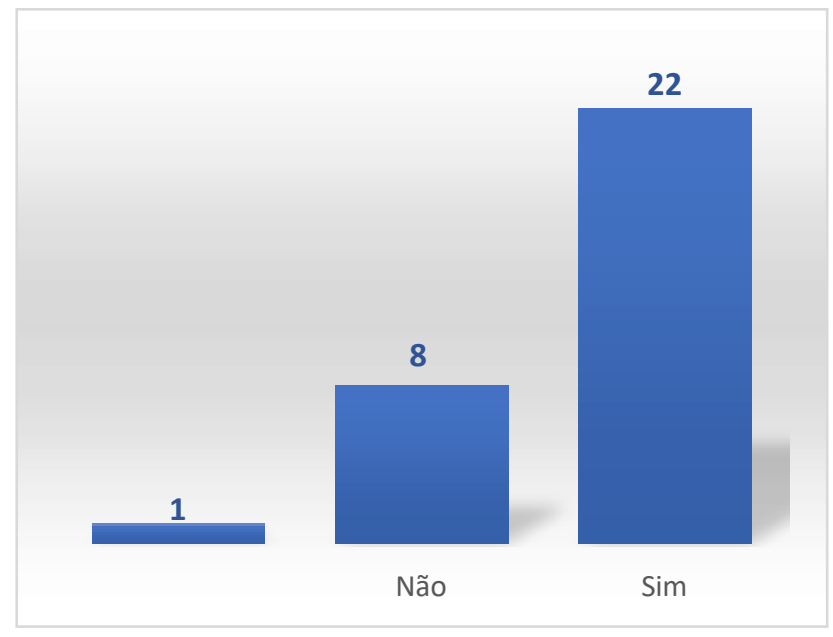

Fonte: Autores (2021).

Para Reis et al. (2017), é importante que os profissionais respeitem o limite de cada mulher, fazendo que o parto seja estabelecido no tempo da mesma, dando a ela todo direito e autonomia sobre seu corpo e como querem que seu parto seja conduzido. Elas podem escolher como querem se posicionar, quais métodos de alivio de dor e o local em que elas vão parir. O gráfico demonstra que essas mulheres reconhecem orientações como solicitação para "fazer força" (Gráfico 7) como normativos durante o parto. Assim como manobras como Kristeller .

Gráfico 7 - Pediram a você para fazer força na hora do parto?

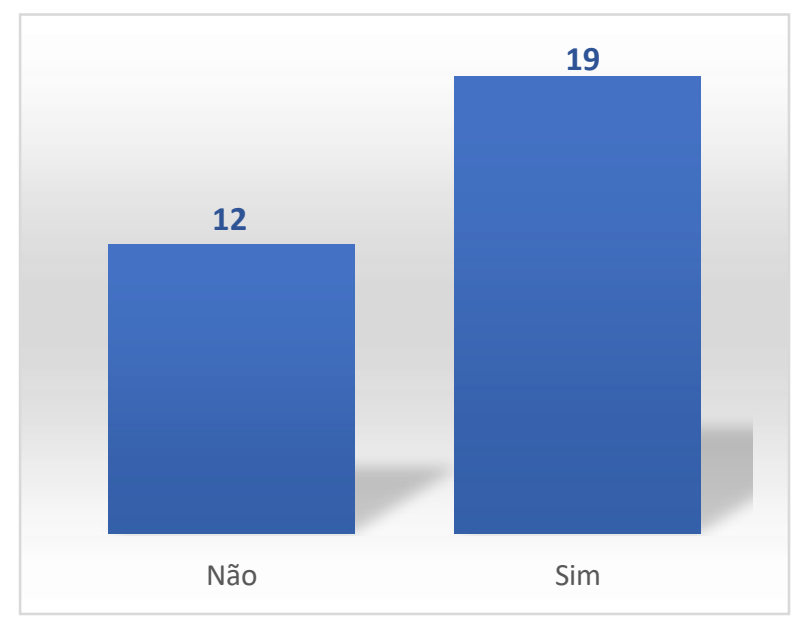

Fonte: Autores (2021).

O Gráfico 8 demostra que as mulheres não perceberam a ocorrência de VO, tal resultado pode estar relacionado ao fato de que as gestantes não tinham a percepção das características do V.O, por falta de informação a respeito dos tipos variados em que se apresentam as diversas facetas da violência em obstétrica. Segundo Araújo e Carvalho (2018), as mulheres desconhecem o que é a violência obstétrica, sendo identificada apenas em momentos que ocorrem como violência verbal e maus tratos, portanto não percebida diante da realização de procedimentos desnecessários. 
Gráfico 8 - Em algum momento você percebeu que vivenciou algum tipo de violência?

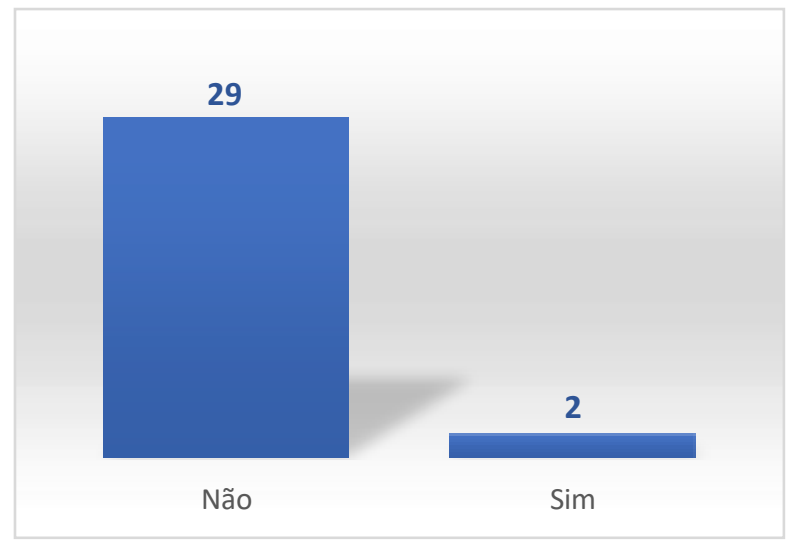

Fonte: Autores (2021).

Durante a entrevista algumas parturientes relataram seus sentimentos e emoções durante o seu processo de parto, dentre eles foram encontradas violências verbais e físicas que a própria gestante desconhece como algo ruim e sim sendo normal.

As puérperas p1, p6, p10, p25, relatam estarem com medo e receio de não conseguir ter o bebê, isso demonstra a falta de preparo que a gestante teve durante seu pré-natal, momento esse que deveria ser usado para esclarecer dúvidas e realizar orientação de todo processo fisiológico materno. A parturiente precisa ser orientada que seu corpo possui uma estrutura fisiológica perfeita para gerar uma gestação, e ter seu bebê.

O sentimento de ansiedade foi relatado pelas pacientes p4 e p23, processo natural durante a gestação devido suas modificações hormonais, o que caracteriza um processo de mudanças constantes nos seus sentimentos, causando assim a ansiedade no trabalho de parto.

A ocitocina é um método farmacológico muito utilizado para aceleração do trabalho de parto, uma vez que o mesmo ajuda a evoluir a fase ativa, aumentar a frequência e intensidade das contrações. Os profissionais fazem uso da medicação sem indicação e muitas vezes rotineiramente, devido sua promessa de diminuir o tempo de processo parturitivo, e assim facilitando seu trabalho e tornando um plantão mais tranquilo. Na paciente p11, foi ofertada medicação com a promessa de "ajudar" nas dores o que não aconteceu, ao contrário da promessa houve aumento nas contrações e violência verbal por parte dos profissionais.

Segundo Andrade (2016), a persistente utilização de práticas não recomendadas pelas evidências científicas, como o uso abusivo de ocitocina, imobilização no leito e posição litotômica no parto, pode levar à compressão de grandes vasos e prolongamento do trabalho de parto (TP) e do período expulsivo e, consequentemente, repercutir negativamente sobre os resultados perinatais.

O toque vaginal gestacional avalia a real dilatação do colo uterino, evolução do trabalho de parto, e eventuais riscos, assim como a bolsa das águas e apresentação fetal, procedimento rotineiro durante a admissão da gestante na maternidade, onde deve ser informado a parturiente a forma de como e realizado o exame e sua real necessidade, porém se faz indispensável reduzir ao mínimo a quantidade de toques para evitar infeções e possíveis traumas nos tecidos maternos. O ato de toque vaginal em excesso é considerado uma prática de violência obstétrica, foi o que aconteceu com a paciente p15, onde a mesma se sentiu "usada" como treinamento para acadêmicos de medicina, gerando sentimento de desrespeito e a traumatizando.

A paciente p21 relatou ter recebido uma ofensa por parte do médico, onde o mesmo minimizou a sua dor do parto e ressaltou, pelo fato da paciente ter tatuagens poderia melhor suportar a dor. Este tipo de conduta é considerado violência obstétrica verbal. 
Não existem evidências que comprovem os benefícios na realização da manobra de Kristeller, muito pelo contrário, devido seus riscos na ruptura uterina, lesões perineais e hemorragias maternofetal esse método e contraindicado, pois, causa danos a mãe e ao bebê. Mesmo sendo um procedimento prejudicial, o mesmo pode ser visto de forma rotineira nas maternidades, com intuito da promessa de antecipar o parto e ajudar o bebê a nascer. Segundo Nascimento et al (2021), todos os relatos analisados em seu estudo demonstram, uma experiência dolorosa e traumática, de cunho psicológico. Caso relatado pelas pacientes p22 e p31, onde subiram e empurraram a barriga da gestante ameaçando a mesma fazer força, caso contrário seu bebê iria morrer.

A lei do acompanhante de $\mathrm{n}^{\circ} 11.108 / 2005$, deve ser garantida em todo o processo de gestacional, o acompanhante pode ser o próprio conjugue, membro familiar ou pessoa de escolha da gestante. Porém, a paciente p26, relata que sua mãe foi orientada a sair da sala pois estava atrapalhando no parto, procedimento desnecessário, pois a acompanhante e uma ancora para suporte emocional da gestante.

\section{Quadro 1.}

\begin{tabular}{|c|c|}
\hline Paciente & Relatos Verbalizados pelas puérperas entrevistadas \\
\hline $\mathrm{P} 1$ & $\begin{array}{l}\text { Assustada por ver mulheres ao meu redor gritando de dor, outras tendo bebê, receio de } \\
\text { não conseguir. }\end{array}$ \\
\hline $\mathrm{P} 4$ & Ansiedade com o trabalho de parto \\
\hline P6 & Medo de não conseguir ter o bebê e do trabalho de parto \\
\hline $\mathrm{P} 10$ & $\begin{array}{l}\text { Por ser meu primeiro filho, eu estava com dúvidas de como seria o trabalho de parto, } \\
\text { estava com medo de não conseguir e ansiedade para ter o bebê nos meus braços e vero } \\
\text { rostinho dele. }\end{array}$ \\
\hline P11 & $\begin{array}{l}\text { O médico me ofereceu um sorinho para ajudar nas dores e trabalho de parto, quando } \\
\text { colocaram o soro comecei a sentir fortes dores e me informaram que era normal e eu } \\
\text { tinha que aguentar porque meu bebê poderia morrer. }\end{array}$ \\
\hline $\mathrm{P} 15$ & $\begin{array}{l}\text { Quando cheguei na maternidade fui atendida e estava cheio de acadêmicos com o } \\
\text { médico que me atendeu. Eu estava com muita dor e o médico foi fazer o toque e depois } \\
\text { cada acadêmico fez o toque. Eles estavam treinando em mi. Eu me senti usada, } \\
\text { desrespeitada. }\end{array}$ \\
\hline $\mathrm{P} 21$ & $\begin{array}{l}\text { Na hora do trabalho de parto eu estava com muita dor, o médico percebeu que eu tinha } \\
\text { tatuagem e disse que eu estava reclamando de dor para ter bebê e com certeza não tinha } \\
\text { reclamado para me tatuar. }\end{array}$ \\
\hline $\mathrm{P} 22$ & Empurraram minha barriga para que o bebê não morresse dentro de mim. \\
\hline $\mathrm{P} 23$ & Ansiedade para ter o bebê \\
\hline $\mathrm{P} 25$ & Medo de não conseguir ter o bebê e que ele morresse na hora do parto. \\
\hline $\mathrm{P} 26$ & $\begin{array}{l}\text { Minha mãe foi orientado a sair da sala de parto pois estava atrapalhando e ela ficou com } \\
\text { medo de acontecer algo comigo e com o bebê }\end{array}$ \\
\hline P31 & $\begin{array}{l}\text { "Teve um momento do meu trabalho de parto que eu já estava sem força e o } \\
\text { enfermeiro subiu na minha barriga e disse " faz força que o seu bebê está morrendo". }\end{array}$ \\
\hline
\end{tabular}

Fonte: Autores (2021). 


\section{Considerações Finais}

O estudo mostra que há questões multifatoriais que acabam contribuindo para a violência obstétrica, por exemplo, as condições sociodemográficas, o não conhecimento acerca da violência obstétrica, pouca escolaridade e outros aspectos que são citadas na pesquisa, há uma necessidade de mudança na assistência e no acolhimento, olhando a parturiente como um todo, respeitado suas emoções e dores durante o pré-parto, parto e pós-parto.

Os profissionais de saúde devem atuar no sentindo de garantir que as mulheres tenham um atendimento digno e respeitoso, preservando os direitos conquistados por elas no campo obstétrico, deste modo as dificuldades de trabalho não podem se sobressaí na hora do atendimento, se tornando como justificativa para casos de violência obstétrica.

Identificamos que há uma necessidade de desenvolvimento de mais estudos que relatem os tipos de violências obstétricas vivenciadas e os fatores que contribuem para a mesma, pois ainda e um assunto que poucas mulheres têm conhecimento, e por isso acabam não sabendo identificar essas condutas perniciosas.

\section{Referências}

Andrade, P. D. O. N., Silva, J. Q. P. D., Diniz, C. M. M., \& Caminha, M. D. F. C. (2016). Fatores associados à violência obstétrica na assistência ao parto vaginal em uma maternidade de alta complexidade em Recife, Pernambuco. Revista Brasileira de Saúde Materno Infantil, $16,29-37$.

Andrade, U. V., Santos, J. B., \& Duarte, C. (2019). A percepção da gestante sobre a qualidade do atendimento pré-natal em UBS, Campo Grande, MS. Revista Psicologia e Saúde, 11(1), 53-61.

Brandão, E. R., \& Cabral, C. D. S. (2021). Juventude, gênero e justiça reprodutiva: iniquidades em saúde no planejamento reprodutivo no Sistema Único de Saúde. Ciência \& Saúde Coletiva, 26, 2673-2682.

Campos, V. S., Morais, A. C., do Nascimento Souza, Z. C. S., \& de Araújo, P. O. (2020). Práticas convencionais do parto e violência obstétrica sob a perspectiva de puérperas. Revista Baiana de Enfermagem34, .

Castro, Antonia Tainá Bezerra; ROCHA, Sibele Pontes. Violência obstétrica e os cuidados de enfermagem: reflexões a partir da literatura. Enfermagem em foco, 11(1).

Coelho, B. S. G., \& Marques, L. C. N. (2016). Violência obstétrica: uma afronta à dignidade da mulher.

Estumano, V. K. C., de Melo, L. G. D. S., Rodrigues, P. B., \& do Rêgo Coelho, A. C. (2017). Violência obstétrica no Brasil: casos cada vez mais frequentes. Revista Recien-Revista Científica de Enfermagem, 7(19), 83-91.

Fernandes, I. B., Bento, P. A. D. S. S., \& Xavier, R. B. (2019). Experiências de mulheres no gestar e parir fetos anencéfalos: as múltiplas faces da violência obstétrica. Interface-Comunicação, Saúde, Educação, 23.

Gabriel, A. D. B., \& Santos, B. R. G. (2020). A Injustiça Epistêmica na violência obstétrica. Revista Estudos Feministas, 28.

Guimarães, L. B. E., Jonas, E., \& Amaral, L. R. O. G. D. (2018). Violência obstétrica em maternidades públicas do estado do Tocantins. Revista Estudos Feministas, 26.

Katz, L., Amorim, M. M., Giordano, J. C., Bastos, M. H., \& Brilhante, A. V. M. (2020). Quem tem medo da violência obstétrica? Revista Brasileira de Saúde Materno Infantil, 20, 623-626.

Lansky, S., Souza, K. V. D., Peixoto, E. R. D. M., Oliveira, B. J., Diniz, C. S. G., Vieira, N. F., ... \& Friche, A. A. D. L. (2019). Obstetric violence: influences of the Senses of Birth exhibition in pregnant women childbirth experience. Ciencia \& saude coletiva, 24, $2811-2824$.

Lamy, Z. C., Gonçalves, L. L. M., Carvalho, R. H. D. S. B. F. D., Alves, M. T. S. S. D. B., Koser, M. E., Martins, M. D. S., ... \& Thomaz, E. B. A. F. (2021). Atenção ao parto e nascimento em maternidades do Norte e Nordeste brasileiros: percepção de avaliadores da Rede Cegonha. Ciência \& Saúde Coletiva, 26, 951-960.

Leal, M. D. C., Bittencourt, S. D. A., Esteves-Pereira, A. P., Ayres, B. V. D. S., Silva, L. B. R. A. D. A., Thomaz, E. B. A. F., ... \& Vilela, M. E. D. A. (2019). Avanços na assistência ao parto no Brasil: resultados preliminares de dois estudos avaliativos. Cadernos de Saúde Pública, 35.

Leal, S. Y. P., de Azevedo Lima, V. L., da Silva, A. F., Soares, P. D. F. L., Santana, L. R., \& Pereira, Á. (2018). Percepção da enfermeira obstetra acerca da violência obstétrica. Cogitare Enfermagem, 23(1).

Marques, B. L., Tomasi, Y. T., Saraiva, S. D. S., Boing, A. F., \& Geremia, D. S. (2020). Orientações às gestantes no pré-natal: a importância do cuidado compartilhado na atenção primária em saúdea. Escola Anna Nery, 25.

Matos, M. G. D., Magalhães, A. S., \& Féres-Carneiro, T. (2021). Violência Obstétrica e Trauma no Parto: O Relato das Mães. Psicologia: Ciência e Profissão, 41. 
Research, Society and Development, v. 10, n. 15, e381101522670, 2021 (CC BY 4.0) | ISSN 2525-3409 | DOI: http://dx.doi.org/10.33448/rsd-v10i15.22670

Martins, F. L., Silva, B. O., Carvalho, F. L. O., Dalmo, M. C., Paris, R. L. P., \& Guidi Junior, L. R. (2019). Violência Obstétrica: Uma expressão nova para um problema histórico. Revista Saúde em Foco, 11, 413-423.

Nascimento, S. L. D., Pires, V. M. M. M., Santos, N. D. A., Machado, J. C., Meira, L. S., \& Palmarella, V. P. R. (2019). Conhecimentos e experiências de violência obstétrica em mulheres que vivenciaram a experiência do parto. Enfermería Actual de Costa Rica, (37), 66-79.

Nascimento, T. L. C., Teixeira, C. S. S., Anjos, M. S. D., Menezes, G. M. D. S., Costa, M. D. C. N., \& Natividade, M. S. D. (2021). Fatores associados à variação espacial da gravidez na adolescência no Brasil, 2014: estudo ecológico de agregados espaciais. Epidemiologia e Serviços de Saúde, 30 , e201953.

Pereira Nery, V., \& Pereira de Lucena, G. (2019). Principais tipos de violências obstétricas sofridas pelas parturientes. Revista Científica de EnfermagemRECIEN, 9(27)

Novellino, M. S. F. (2011). Um estudo sobre as mães adolescentes brasileiras. Physis: Revista de Saúde Coletiva, 21, $299-318$.

Ramos C. A. C. (2020). A responsabilidade civil por violência obstétrica: O caso da episiotomia no Brasil.

Reis, T. L. D. R. D., Padoin, S. M. D. M., Toebe, T. R. P., Paula, C. C. D., \& Quadros, J. S. D. (2017). Autonomia feminina no processo de parto e nascimento: revisão integrativa da literatura. Revista Gaúcha de Enfermagem, 38.

Rocha, N. F. F. D., \& Ferreira, J. (2020). A escolha da via de parto e a autonomia das mulheres no Brasil: uma revisão integrativa. Saúde em Debate, 44, 556568.

Santos, H. F. L., \& Araujo, M. M. (2016). Políticas De Humanização Ao Pré-Natal E Parto: Uma Revisão De Literatura Humanization the Policies Prenatal and Childbirth: a Literature Review. Revista Científica FacMais, 6(2), 54-64.

Silva, L. N. M. D., Silveira, A. P. K. F., \& Morais, F. R. R. D. (2017). Programa de humanização do parto e nascimento: aspectos instituicionais na qualidade da assistência. Rev. enferm. UFPE on line, 3290-3294.

Silva, M. R. C. D. (2019). Dor e violencia no parto: maus tratos a mulheres em uma maternidade de Manaus.

Souza, S. D. O. D., Mangueira, T. R. E., \& Cordeiro, W. (2020). Violência obstétrica no Brasil: uma análise acerca da violação à ética médica e à dignidade humana Obstetric violence in Brazil: an analysis on violation of medical ethics and human dignity. Rev. Bras. de Direito e Gestão Pública (Pombal, PB), 8(03), 889-904.

Tobar, J. E., Cuchumbe-Sánchez, Y. D., Ledesma-Rengifo, J. B., Muñoz-Mosquera, M. C., Campo, A. M. P., \& Suarez-Bravo, J. P. (2019). Violencia obstétrica: haciendo visible lo invisible. Revista de la Universidad Industrial de Santander. Salud, 51(2), 135-146.

Tomasi, Y. T., Saraiva, S. D. S., Boing, A. C., Delziovo, C. R., Wagner, K. J. P., \& Boing, A. F. (2021). Do pré-natal ao parto: um estudo transversal sobre a influência do acompanhante nas boas práticas obstétricas no Sistema Único de Saúde em Santa Catarina, 2019. Epidemiologia e Serviços de Saúde, 30.

Thiry-Cherques, H. R. (2009). Saturação em pesquisa qualitativa: estimativa empírica de dimensionamento. Revista PMKT, 3(2), $20-27$.

Vicente, A. C., Lima, A. K. B. S., \& Lima, C. B. Parto cesário e parto normal: uma abordagem acerca de riscos e benefícios. Temas Saúde. 2017 [cited 2020 Feb 24]; 17 (4): 24-35.

Zanardo, G. L. D. P., Uribe, M. C., Nadal, A. H. R. D., \& Habigzang, L. F. (2017). Violência obstétrica no Brasil: uma revisão narrativa. Psicologia \& sociedade, 29. 